\title{
Detecting nonlinearity in psychological data: Techniques and applications
}

\author{
RICHARD A. HEATH, ALICE KELLY, and MITCHELL LONGSTAFF \\ University of Newcastle, Callaghan, New South Wales, Australia
}

\begin{abstract}
Modern graphical and computational techniques for detecting nonlinearity in psychological data sets are presented. These procedures allow researchers to determine the information complexity of temporal data, using physiological and psychological measurements, and to provide evidence for chaos in time series contaminated by measurement noise. Problems with noise reduction and appropriate experimental control, using surrogate time series, are discussed, and applications of the technology are illustrated, using response time, handwriting, and typing data sets. In an experimental application of appropriate nonlinear analysis procedures, the results of a time series prediction experiment confirm that some subjects are sensitive to chaos. In contrast to previous attempts demonstrating sensitivity to chaos, the experiment reported here employs surrogate series to control for linear stochastic aspects of the stimulus sequences, such as autocorrelation. Recommendations for the selection of appropriate software for performing nonlinear analyses are presented, including a comprehensive list of WorldWide Web sites offering such software.
\end{abstract}

Most statistical analysis procedures used by psychologists, such as analysis of variance and discriminant analysis, are based on the general linear model or one of its multivariate generalizations. Except for repeated measures designs, with their attendant constraints, such as compound symmetry (Howell, 1997), most of these conventional analyses do not incorporate an important temporal element. Most people would intuit that human behavior is observed in a spatiotemporal environment in which the primary measurements are multivariate and temporal. It is surprising, therefore, that very little psychological research employs the interesting and challenging methodology of time series analysis.

Conventional time series analysis represents time either as essentially continuous, as in the record traces for physiological measures, such as heart rate and EEG, or as discrete events, evenly or, frequently, unevenly spaced in time. The primary measurement tools include autocorrelations that relate behavior sampled at different time lags. Intuitively, behavior is more highly related (i.e., correlated) the more temporally contiguous it is. This principle, of course, is the mainstay of numerous theories of learning, conditioning, and memorization. As the temporal separation between events increases, the autocorrelation frequently decreases, leading to a violation of the compound symmetry assumption.

This research was supported by a research grant under the Australian Research Council Small Grants Scheme to R.A.H. and A. Heathcote. We thank Nadine Smith for assisting in the running and analysis of the chaos prediction experiment. Correspondence concerning this article should be addressed to R. A. Heath, Department of Psychology, University of Newcastle, University Drive, Callaghan, NSW 2308, Australia (e-mail: heath@psychology.newcastle.edu.au).
It is worthwhile noting that spectral analysis, a commonly used measurement procedure in physiological psychology, encapsulates temporal information by transforming time into frequency. A related technique regresses the current observation in a behavioral time series on previous values from the same series to predict future behavior. This process yields linear autoregression equations (Gottman, 1981) that have mathematical properties similar to those of the more familiar multiple linear regression equations derived from the general linear model. In the temporal case, we predict the next observation from the same (hence, auto) observation set, whereas in multiple linear regression, the criterion is generally quite different from any of the predictors.

Most psychologists are familiar with the general linear statistical model in the guise of multiple linear regression and its special case, analysis of variance and covariance. However, many psychological phenomena deviate somewhat from the linear model's predictable, gradual change over time. What is frequently significant to both casual and professional observers of human behavior, whether it be normal or psychopathological, is the sudden and almost unpredictable changes that can occur. Any quantitative analysis of such changes requires the consideration of a nonlinear model, one that does not follow the steady predictable change that characterizes linear representations.

Many scientists, psychologists included, have become increasingly intrigued by the rich behavior representations that are possible with nonlinear methods. By simply changing the value of a single parameter in an equation, such as the logistic recursion (Kaplan \& Glass, 1995), we can generate behavior sequences that converge on either one state or an ordered sequence of stable states. These are known as fixed-point and limit cycle attractors, respectively. Under other conditions we can generate 
chaotic behavior that appears noiselike but is actually deterministic and predictable in the short term. Even more intriguing is the prospect of estimating a precise parameter range where the nonlinear system spontaneously self-organizes and exhibits lifelike behavior. This; of course, is the controversial edge-of-chaos phenomenon (Heath, 1998; Kauffman, 1993).

This paper is designed to familiarize psychologists with a selection of up-to-date methods for evaluating nonlinear dynamics in behavior sequences. Basic computation procedures will be accompanied by specialized techniques for detecting nonlinear determinism in noisy experimental data, together with hypothesis-testing procedures that use surrogate data sequences. The paper concludes with some illustrative applications of the techniques in a variety of behavioral tasks, including a unique procedure for evaluating human sensitivity to chaos.

\section{Estimating Nonlinear Determinism \\ From Behavior Sequences}

Data suitable for nonlinear analysis consist of the sequential observation of a behavior characteristic, such as a mood, at regular intervals or as a series of time measures, such as response time (RT) or typing interkeypress time (IKT). The latter can be converted into a response rate and then interpolated so that the sampling over time is regular. Otherwise the actual time delays can be simply mapped onto the integers so that the $n$th RT, RT $(n)$, is treated as if it were observed at equally spaced points in time, roughly equal to the total observation period divided by the total number of observations, $N$.

Other possibilities exist when there are simultaneous recordings, as with a multichannel EEG. In such a case, there can be nonlinear processes within a recording channel, as well as complex interactions between channels. Linear methods that include commonly employed spectral analyses are not designed to cater to such complexities.

Consider a sequence of several thousand IKTs recorded during a single typing session lasting at least $30 \mathrm{~min}$. First, any linear (or possibly, second-order polynomial or above) trend is removed from the data sequence, and the resulting residuals are analyzed. A preliminary linear analysis reveals that an autoregression equation of the form

$$
\operatorname{IKT}(n+1)=\sum_{i=1}^{r} a_{i} \operatorname{IKT}(n+1-i)
$$

fits the data adequately, for sections of the data that are stationary - that is, the autoregression parameters do not change over time. When the data are nonstationary, it may be necessary to fit different autoregression equations to stationary nonoverlapping partitions of the time series.

Evidence for nonlinear determinism in the detrended IKT time series can be obtained by controlling for all of the linear dynamics present in the time series. Thanks to modern nonlinear data analysis technology, this apparently impossible process is less difficult than it may seem at first. Physicists, such as Abarbanel (1996) and Kantz and Schreiber (1997), who have at hand long time series from possibly chaotic stationary sources, have devised techniques for transforming data sequences into samples containing all of the linear information-that is, autocorrelation and corresponding power spectra - in the original data set. These samples are known as phase-randomized Gaussian-scaled surrogate series. They are created by applying Fourier analysis, randomly scrambling the phase spectrum, and then reconstructing the time series, using both the original power spectrum and the new phase spectrum. The resulting time series is then rescaled so that it conforms with a Gaussian distribution. This means that samples from a null hypothesis population of equivalent linear processes are available so that a nonlinear quantifier, computed from the experimental series, can be evaluated. If the experimental value lies outside of the $(1-\alpha) 100 \%$ confidence interval for this same statistic under the null hypothesis, we can reject the latter and conclude that the time series dynamics are not entirely linear. Various converging analyses can then be implemented to determine the exact nature of the nonlinear dynamics.

The decision process can be summarized as follows. Decide on a nonlinear quantifier, such as the time reversal index or the correlation dimension (Heath, 2000), and then determine its distribution for surrogate series that have all of the linear properties of the experimental time series but none of the nonlinear dynamics. If the same statistic computed for the experimental series is not contained in this confidence interval, reject the null hypothesis and conclude that the experimental series has nonlinear determinism. In the next section, we will examine the results of several such analyses, using IKT time series $(N=2,480$; Heath \& Willcox, 1990), sequential RTs recorded in a four-choice task $(N=4,800$; Kelly, Heathcote, Heath, \& Longstaff, in press), and a horizontal handwriting velocity time series $(N=3,338$; Longstaff \& Heath, 1999).

\section{Linear Properties of the Experimental Time Series}

The linear properties of the experimental time series, $X(t), t=1, N$, can be best represented by lagged phase plots, which suggest the presence of autocorrelation in the data set, as well as power spectra. On such plots, $X(t)$ is plotted against $X(t-L)$, when the lag is $L$, or alternatively, an estimate of the derivative of the time series at time $t$. All the surrogate series are constructed so that every one of these linear properties is preserved.

The lag one phase plots presented in Figures 1A, 2A, and $3 \mathrm{~A}$, for the IKT, RT, and handwriting velocity time series, respectively, do not clearly indicate nonlinear determinism, although the plot for the handwriting velocity data appears to be the most highly structured of the three. The general positive slopes for both the RT and the handwriting velocity time series indicate significantly positive lag one autocorrelation for these series. The corresponding power spectra, shown in Figures 1B, 2B, and $3 \mathrm{~B}$, are reasonably structured, with most energy being located at the lower frequencies. It is noteworthy that the spectra for both the experimental and a sample surrogate series, constructed using phase randomization, are iden- 

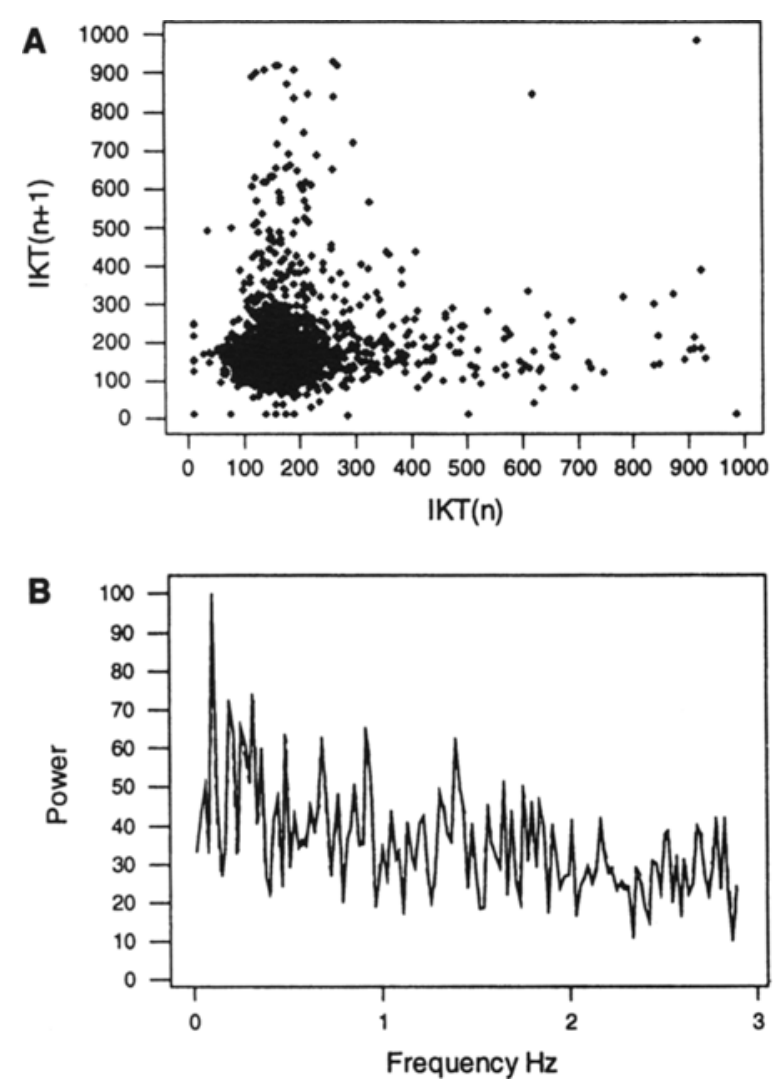

Figure 1. (A) IKT Lag 1 phase plot for interkeypress time (IKT) data for Subject 10. All IKTs greater than 1,000 msec have been omitted. (B) Power spectrum for IKT data for Subject 10. The spectra for the experimental (continuous line) and a representative surrogate series (broken line) are superimposed.

tical. This observation confirms that each surrogate series contains all of the linear structure contained in the corresponding experimental time series. So, the distribution of indices for the surrogates enables the null hypothesis of linearity to be evaluated, using a resampling scheme that provides an estimate of the sampling distribution when the null hypothesis is true.

\section{Testing the Null Hypothesis of Linearity for a Time Series Sample}

A linear system, such as an undeformable elastic object dropping within a vacuum under the force of gravity and then bouncing up to reach the same height above ground level, has the interesting property that its dynamics remain invariant under reversal of the time dimension. In other words, a video of the phenomenon will look identical to the observer whether it is played forward or backward. The same cannot be reported for irreversible, possibly nonlinear systems, for which the dynamics are distinctly time irreversible. An example of this time reversibility is an Australian road safety advertisement that shows a car accident in which a car rolls over several times and apparently kills the driver. A reversed video then reconstructs the happy situation that existed prior to the accident. To the observer, this time reversal is bizarre and, of course, impossible in real life. So a useful nonlinear measure involves computing a time asymmetry index, using (Schreiber, 1999)

$$
\phi^{\mathrm{rev}}(\tau)=\frac{\sum_{n=\tau+1}^{N}[X(n)-X(n-\tau)]^{3}}{\left\{\sum_{n=\tau+1}^{N}[X(n)-X(n-\tau)]^{2}\right\}^{\frac{3}{2}}} .
$$

Since the time asymmetry index requires a reversible sign, it is computed as the cube of the difference between time series observations with a lag of $\tau$ time units divided by the cube of the root sum of squares of the same difference. This procedure yields an appropriate scalar time asymmetry index.

The results of this analysis, pooled over the various values of lag, $\tau$, can be computed with the TISEAN (Hegger, Kantz, \& Schreiber, 1999) timerev command. The strategy involves estimating the time asymmetry index, $\phi_{\mathrm{rev}}$, for the experimental series and then computing the same index
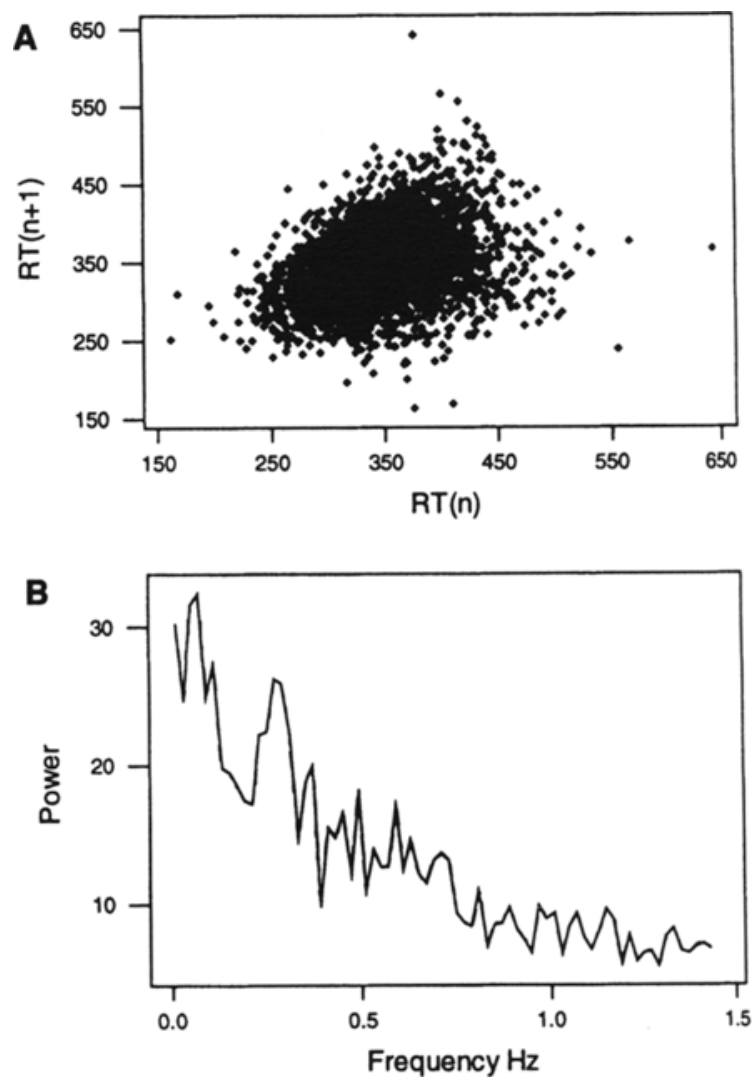

Figure 2. (A) Lag 1 phase plot for sequential response time (RT) data for Subject 3. (B) Power spectrum for RT data for Subject 3. The spectra for the experimental series (continuous line) and a representative surrogate series (broken line) are superimposed. 

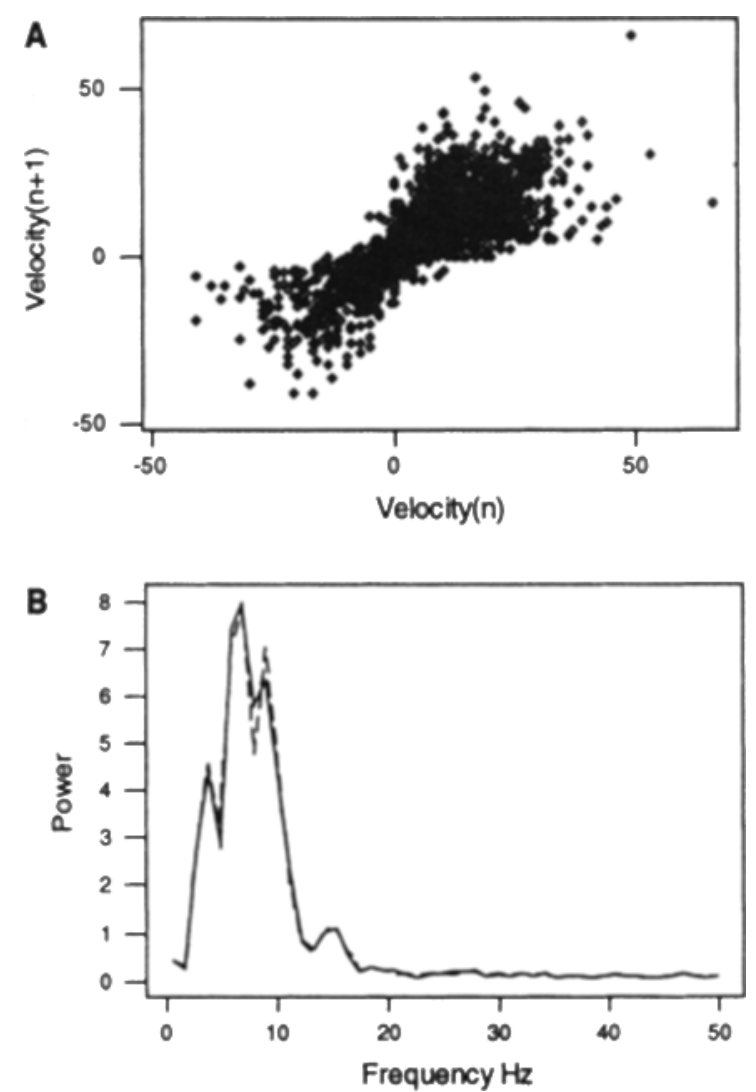

Figure 3. (A) Lag 1 phase plot for handwriting velocity data for Subject 11. (B) Power spectrum for handwriting velocity data for Subject 11. The spectra for the experimental series (continuous line) and a representative surrogate series (broken line) are superimposed.

for a moderately large number of surrogate series, generated using the TISEAN surrogates command. These latter series are generated from the experimental series and have all of the linear properties of that series, including the mean, variance, autocorrelation, and power spectrum.

The TISEAN surrogates command can also ensure that the distribution of data values remains invariant between the experimental and the surrogate series, although some compromise between equality of distributions and equality of power spectra needs to be considered. All that is missing from the surrogate series is the nonlinear determinism, which is removed by randomizing the phase spectrum prior to reconstruction of the surrogate series. So the null hypothesis of linearity can be evaluated, using the distribution of the $\phi_{\mathrm{rev}}$ statistic for all of the surrogate series. Provided the sample size exceeds, say, 30 , the test statistic

$$
z=\frac{\phi_{\exp }-E\left[\phi_{\text {surr }}\right]}{\sqrt{\operatorname{Var}\left[\phi_{\text {surr }}\right]}}
$$

is distributed approximately as a standardized normal distribution. $\phi_{\exp }$ is the sample estimate of the time asymmetry parameter for the experimental series, and $\phi_{\text {surr }}$ is the estimate of the corresponding parameter for the surrogate series. $E\left[\phi_{\text {surr }}\right]$ and $\operatorname{Var}\left[\phi_{\text {surr }}\right]$ are the mean and variance of $\phi_{\text {surr }}$, respectively.

As is shown in Figures 4, 5, and 6, the time asymmetry index for the experimental data series was not significantly different from the index computed for the corresponding surrogate series for the IKT data $(z=-1.25, p>.05)$, but it was significantly different for both the RT sequence data $(z=6.67, p<.001)$ and the handwriting velocity data $(z=5.71, p<.001)$. This result suggested that, whereas the IKT series had no discernible nonlinear determinism, the other two series did.

\section{A Reevaluation of Nonlinear Determinism, Using Noise Reduction}

Experimental data are frequently affected by both measurement noise and noise from other sources, such as stochastic aspects of the process dynamics. Consequently, any nonlinear determinism can frequently be difficult to

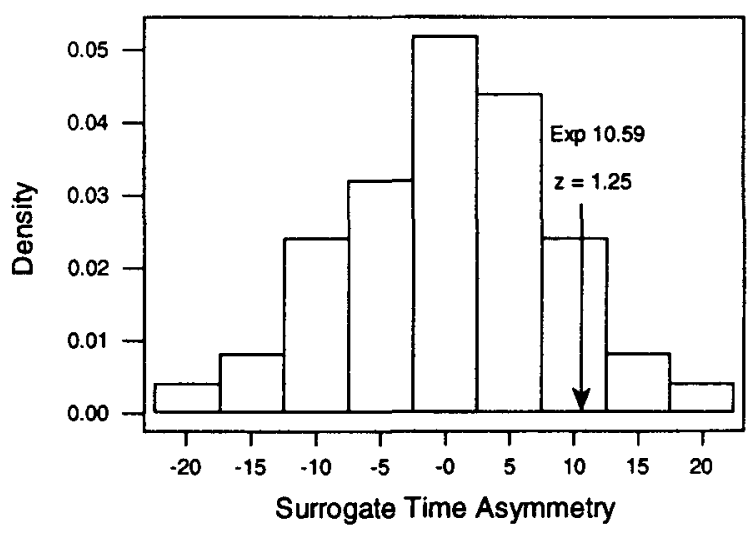

Figure 4. Time asymmetry test distribution for the surrogate series generated from the interkeypress time data for Subject 10. The index for the experimental series is indicated by the arrow.

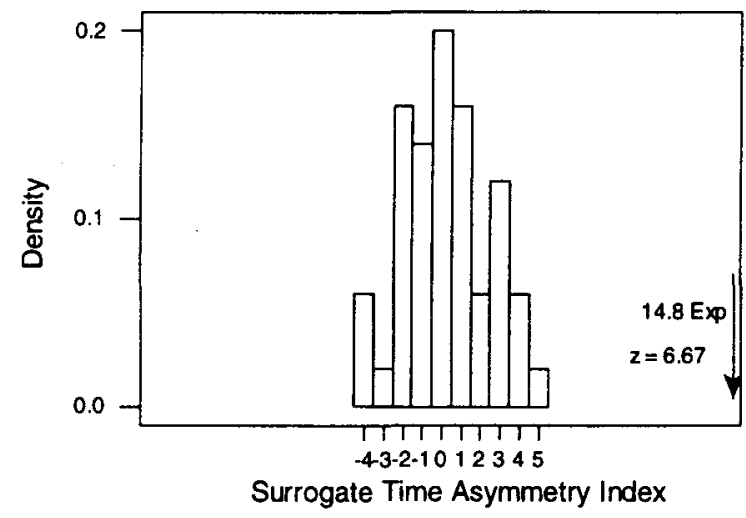

Figure 5. Time asymmetry test distribution for surrogate series generated from the response time sequence data for Subject 3 , using a four-choice task. The index for the experimental series is indicated by the arrow. 


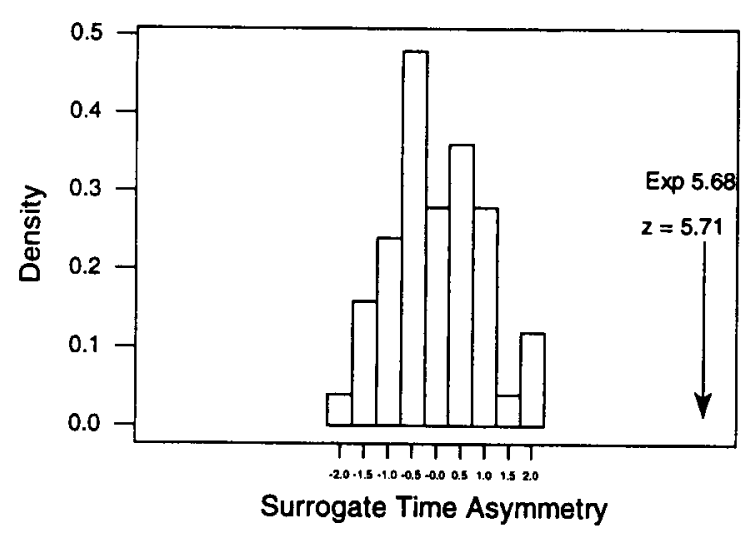

Figure 6. Time asymmetry test distribution for surrogate series generated from the handwriting velocity data for Subject 11. The index for the experimental series is indicated by the arrow.

detect without some form of noise reduction. The techniques for reducing noise in experimental time series involve first transforming the unidimensional time series into points located in a suitably determined multidimensional space, so that (1) each dimension of the space is independent and (2) the number of dimensions is sufficiently large that interpoint distances are consistent throughout the series. Once the first condition is satisfied, we have an embedding, and the second condition ensures that the number of dimensions is sufficiently large that the proportion of falsely located nearest neighbors is negligible. For example, if a sphere is collapsed from three to two dimensions, points at the poles will become nearest neighbors, whereas in an adequate number of dimensions (three in this case), they are maximally distant.

Noise reduction was effected by using the TISEAN program project, which assumes that the experimental data are represented by a low-dimensional system containing the nonlinear deterministic signal and a high-dimensional component representing noise (Grassberger, Hegger, Kantz, Schaffrath, \& Schreiber, 1993). The algorithm employs orthogonal projections of the original data set onto the lower dimensional space to enhance the signal-noise ratio over several iterations of the procedure. This process tends to reduce the data variability over successive applications of the algorithm in a fashion similar to principal components analysis - that is, the first component accounts for most of the variability, and successive components account for decreasing proportions of the remaining variability.

The RT sequence data were embedded in four dimensions, projected along a single phase space dimension, with averaging occurring over 100 nearest neighbors, using a minimum nearest neighbor radius of 0.1 . Five iterations were performed to reduce the standard deviation from 43.8 for the original data to 23.9 following noise reduction. The effect of noise reduction can be seen in Figures 7A and 7B, where the noise-reduced graph reveals a greater level of structure than is evident in the plot of the original data.

A similar revelation of structure following noise reduction is evident in Figures $8 \mathrm{~A}$ and $8 \mathrm{~B}$ for the handwriting velocity data for Subject 11 . Using the TISEAN project command and the same parameters as those used for the RT sequence data, the standard deviation was reduced from 11.02 to 9.23 . When compared with the RT data, the handwriting phase plots became more highly structured following noise reduction.

In the following analyses, the noise-reduced series are used, on the basis of the assumption that the nonlinear determinism, if present, serves as a signal to be detected in the presence of stochastic noise. By reducing the latter, there is a greater opportunity to quantify the nonlinear structure.

\section{Using Recurrence Plots to Detect Nonlinear Determinism}

A recurrence plot prints a series of colored points on a graph containing identical $x$ - and $y$-axes, each axis representing the numerical sequence of the $N$ data values in the time series. The color of the point $(i, j), i, j=1, N$, is proportional to the distance between these points when they are embedded in an appropriate embedding space.
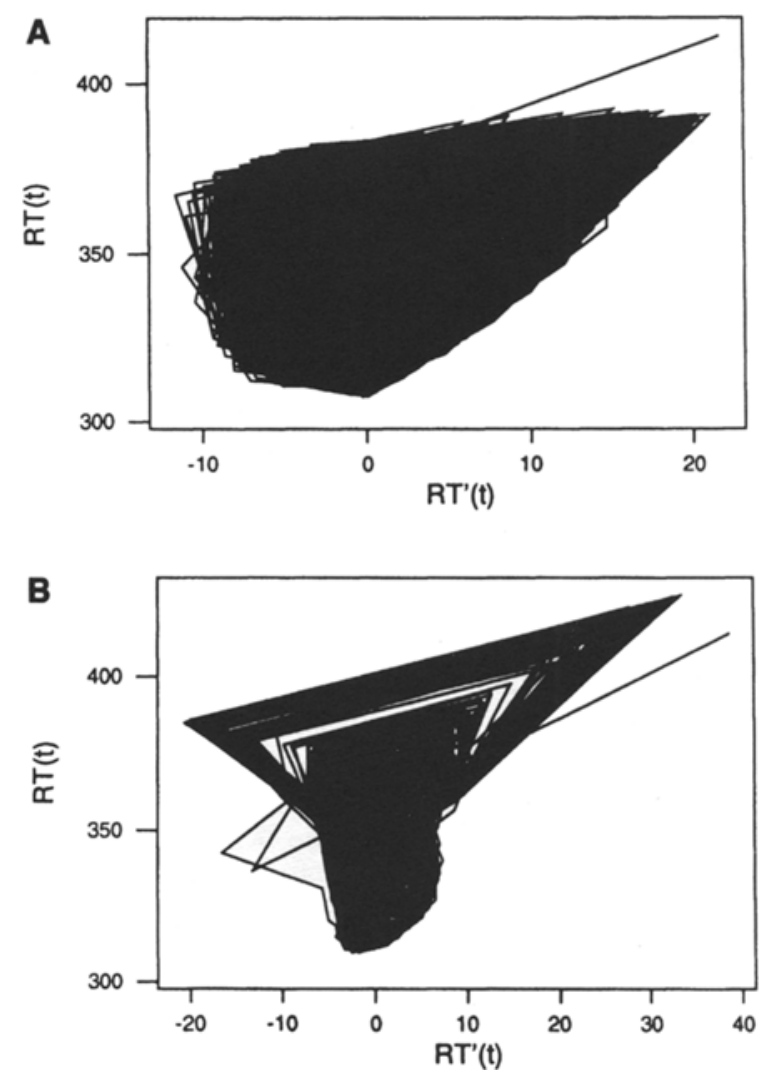

Figure 7. Original (A) and noise-reduced (B) phase plots for the response time (RT) sequence data for Subject 3. 

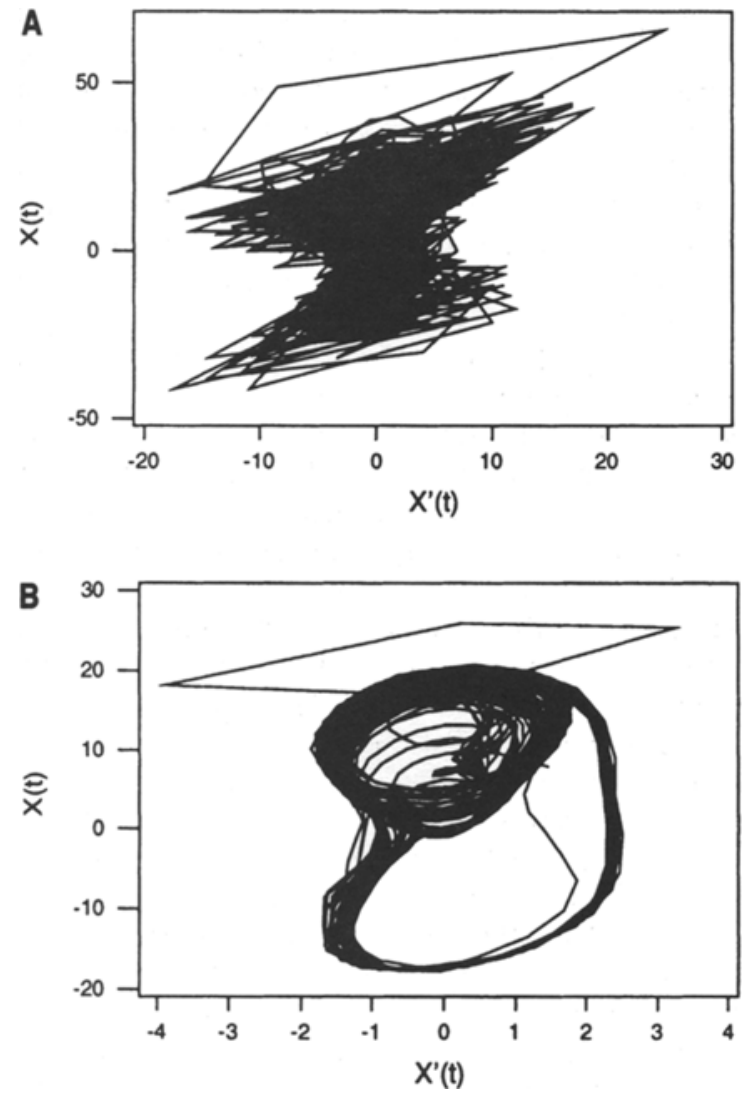

Figure 8. Original (A) and noise-reduced (B) phase plots for the handwriting velocity sequence data for Subject 11.

The coordinates of the embedded points are selected so that each successive coordinate is uncorrelated. The dimensionality of the space is determined by ensuring that the proportion of false nearest neighbors is small.

The recurrence quantification analysis indices were computed with a minimum diagonal line of contiguous recurrent points (suggestive of nonlinear determinism) being set equal to 3, a Euclidean metric being used to compute interpoint distances. The minimum radius for recurrence of neighboring points was set equal to 10 . These plots were obtained with the Visual Recurrence Analysis program written by Eugene Kononov and based on the procedures outlined in Webber and Zbilut (1996).

Both the noise-reduced RT series and its surrogate were embedded in 10 dimensions with a delay, or lag, of 9 . The recurrence plots for these series are depicted in Figures $9 \mathrm{~A}$ and $9 \mathrm{~B}$, respectively. The values of \%recurr, the percentage of points that are recurrent - that is, sufficiently close (less than 10 units) to each other in the plot - of $\%$ deter, the percentage of lines that contain 3 contiguous points, and of entropy, a measure of how structured the recurrence plot is, were computed. There was scarcely any difference between the $\%$ recurr (.03\% and $.04 \%$, respectively) for the experimental and surrogate series, but \%deter was much larger for the experimental series $(41.8 \%$ vs.
$0.91 \%$ ). The experimental series was also more highly structured than the surrogate series, with entropy of 2.19 and 0.65 bits, respectively. Altogether, these quantitative indices, derived from the recurrence analysis, suggest that there is nonlinear determinism in the RT time series.

Figures 10A and 10B depict the recurrence plots for the handwriting velocity data for the noise-reduced and surrogate series, respectively. The quantitative indices were quite similar for the experimental and surrogate series $(\%$ recurr $=3.6 \%$ and $3.8 \%$, \%deter $=69.4 \%$ and $60.6 \%$, and entropy $=3.16$ and 2.52 ). There was just a

A

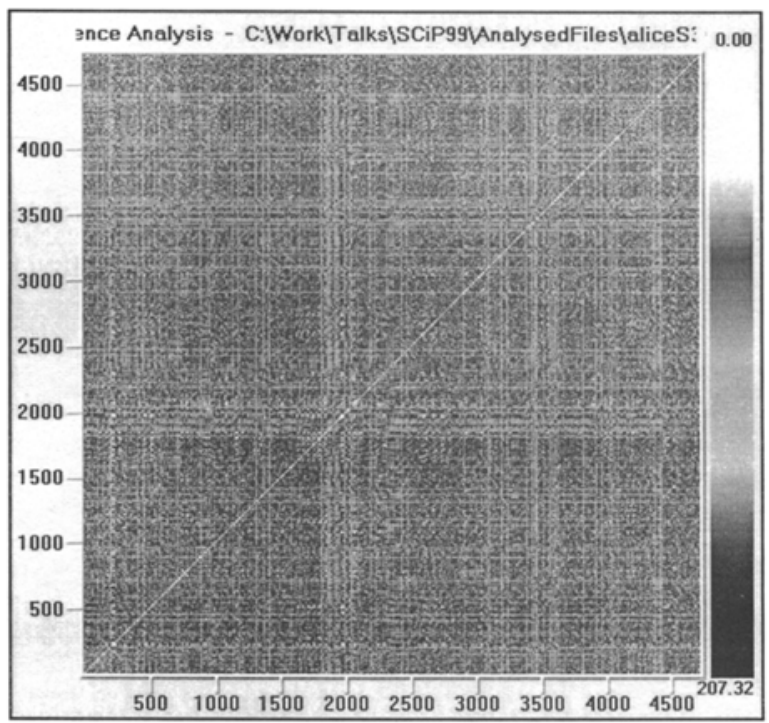

B

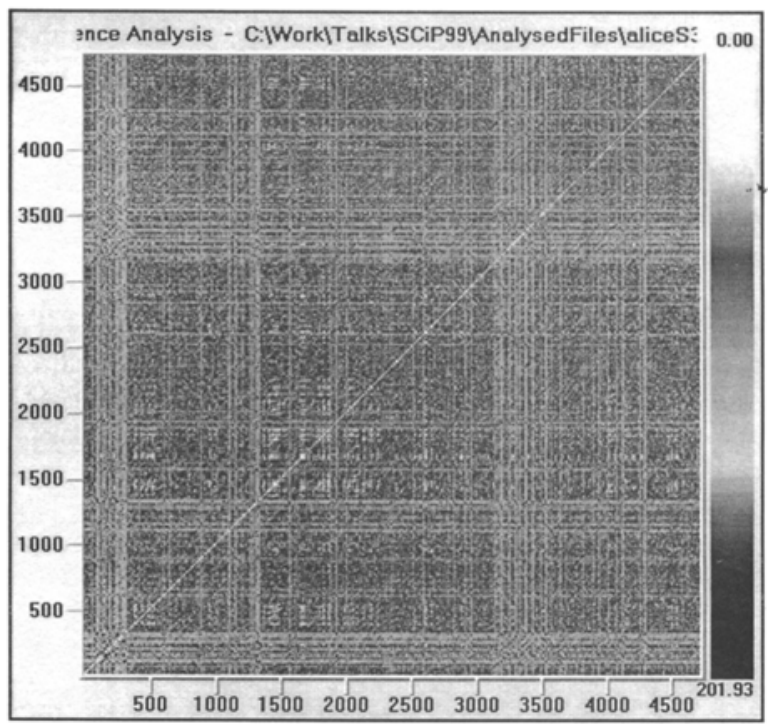

Figure 9. Recurrence plot for response time sequence data (A) and the corresponding surrogate series $(B)$ for Subject 3 . Both series were embedded in 10 dimensions, with a delay of nine. The minimum length of diagonal lines was three. 
hint that there might be nonlinear determinism, but the evidence was not as strong as it was for the RT series.

\section{Nonlinear Prediction}

Chaos in experimental time series can be detected by comparing the prediction plots for the experimental and the corresponding surrogate data sets. When autocorrelation is present in the time series, the prediction accuracy decreases steadily over future time. A similar result also applies when chaos is present, since sensitive dependence on initial conditions, a fundamental property of a

\section{A}

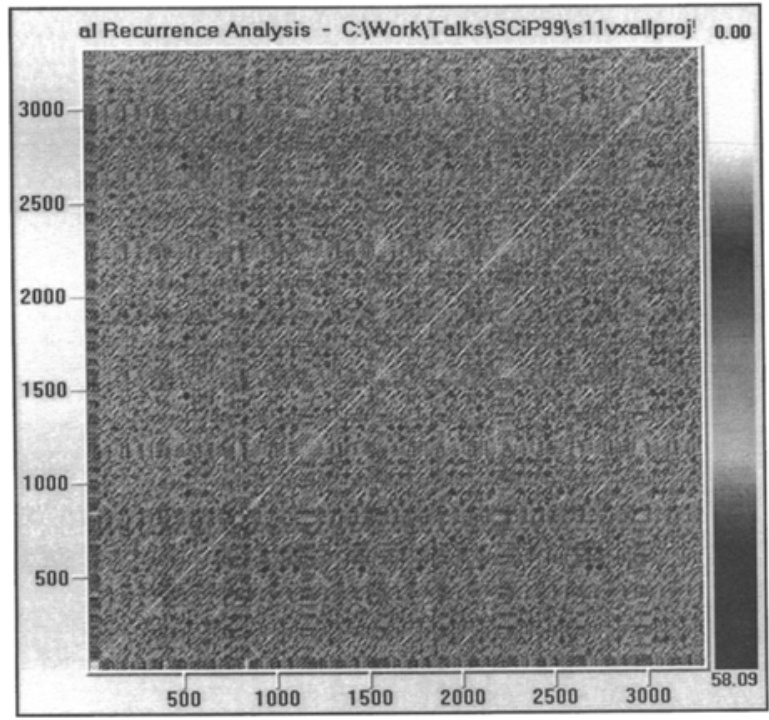

B

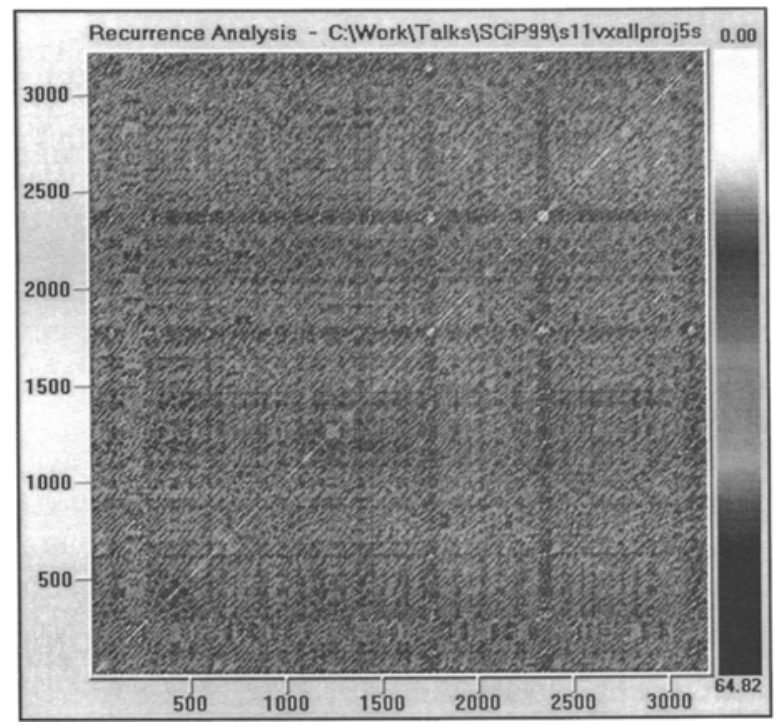

Figure 10. Recurrence plot for handwriting velocity data $(A)$ and the corresponding surrogate series (B) for Subject 11. Both series were embedded in three dimensions, with a delay of 10 . The minimum length of diagonal lines was three. chaotic system, implies that prediction error should increase steadily over time. In view of the similar effects generated by autocorrelation and chaos, appropriate surrogate series are needed to control for linear aspects of the data set.

Figure 11 shows the relative prediction error (RPE)that is, the mean square prediction error divided by the standard deviation - for the RT series. The TISEAN program predict was used for these analyses. This program simply averages the future observations within a small set of nearest neighbor points and uses this average as the prediction one step ahead in time. This process is continued one step at a time to predict the time series for larger temporal windows. It is clear from the figure that RPE increases steadily with prediction lag for both the experimental and the surrogate series but is greater for the surrogate series. In this case, the delay was set equal to one, the number of embedding dimensions was four, and the neighborhood radius was five. This result is consistent with the proposition that the RT sequence data contain nonlinear determinism and, possibly, chaos.

A similar analysis of the handwriting velocity data, shown in Figure 12, indicated that the RPE increased steadily with prediction lag for both the noise-reduced experimental and the surrogate series. Whereas the surrogate series asymptotes at around 1.0, the experimental series does not reach asymptote within the number of prediction lags used. This result suggests that the handwriting velocity time series contains some nonlinear determinism, accompanied possibly by a long-term autocorrelation structure within the time series.

\section{Application of Surrogate Data Technology to Experimental Design}

Several investigators have shown that people can predict chaotic sequences at better than chance (Metzger, 1994; Metzger \& Theisz, 1994; Neuringer \& Voss, 1993; Smithson, 1997). However, it is still uncertain whether these people are indeed relying on the nonlinear determinism in the time series or whether their predictions are facilitated by the linear autocorrelation in the series. This autocorrelation allows the next observation in the series to be predicted by using a linear function of a finite number of previous observations. Since such predictions can decline in accuracy with an increase in the look-ahead window, a similar decline in human prediction accuracy cannot be diagnostic of chaos sensitivity.

Nevertheless, we can use surrogate series technology to overcome this problem. Rather than employing randomly shuffled series as a control, in the proposed methodology, phase-randomized surrogate series are used. In the experimental task, subjects were provided with the previous 8 days' temperatures (in a range from $10^{\circ} \mathrm{C}$ to $30^{\circ} \mathrm{C}$ ) and were required to predict the temperatures for the next 4 days, a task with some face validity in media weather forecasting. The time series for the experimental group was a sample from a stationary chaotic Hénon attractor, and the control group experienced a sample from the phase-randomized surrogate series correspond- 


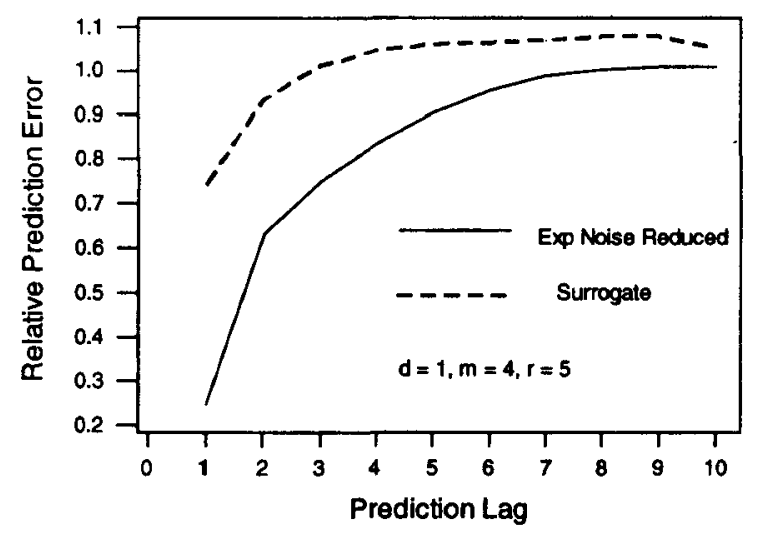

Figure 11. Nonlinear prediction plots for the response time sequence data for Subject 3. The noise-reduced series result (solid line) is compared with the noise-reduced surrogate (broken line) series, using a delay of one, four embedding dimensions, and a nearest neighbor radius equal to five.

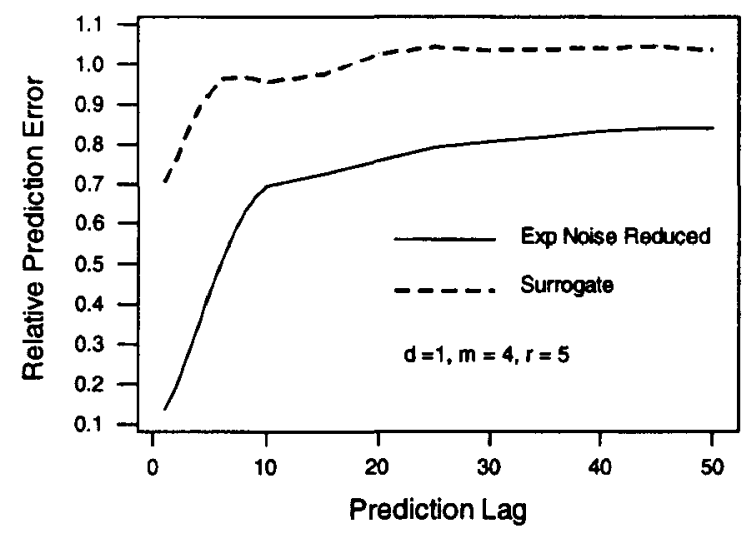

Figure 12. Nonlinear prediction plots for the handwriting velocity data for Subject 11. The noise-reduced series result (solid line) is compared with the noise-reduced surrogate (broken line) series, using a delay of one, four embedding dimensions, and a nearest neighbor radius equal to five.

ing to the Hénon attractor (see Heath, 2000, for an account of these chaotic series). The time series values were linearly transformed to provide "temperatures" in an appropriate range.

Each subject estimated the four temperatures on each of 120 trials. The data shown in Figure 13 are the mean RPE $\left(1-r^{2}\right)$ plotted against days, where $r$ is the Pearson correlation between the observed and the predicted temperatures on that day, for both the Hénon chaotic time series and the surrogate series. The interaction between the days and the series factors was statistically significant $[F(3,25)=6.34, p=.001]$, demonstrating that prediction accuracy for the chaotic time series declined steadily over time, whereas prediction was not possible for the surrogate series. This result suggests that some people are sensitive to chaos. So the methodology used to detect nonlinear determinism and, possibly, chaos in experimental time series also provides a methodological tool for detecting sensitivity to chaos in human decision makers.

\section{Evidence for Chaos in Experimental Data}

The time reversibility and nonlinear prediction tests have supported the proposal that the RT and handwriting velocity series are nonlinear deterministic and, possibly, chaotic. In order to confirm chaos, we need to estimate the full Lyapunov spectrum that estimates the exponential rates of expansion (positive Lyapunov exponent) and contraction (negative Lyapunov exponent) of nearby points in orthogonal directions within the attractor. Chaos is evident when at least one of the Lyapunov exponents is positive (local instability) while their sum is negative (global stability).

The full Lyapunov spectrum can be estimated for relatively short experiments, using the NETLE software (Gençay \& Dechert, 1992). As was described in Heath (2000), the program fits a nonlinear function predicting the current time series value from $w$ previous values, using a feed-forward neural network. The window parameter, $w$, is the dimensionality of the space within which the series is embedded.

The Lyapunov exponent estimates were computed for the noise-reduced $\mathrm{RT}$ and handwriting velocity time series and their corresponding surrogate series, using a fourdimensional embedding space (four input units), six hidden units, and a one-dimensional output. This neural network provided the best fit to the nonlinear time series in each case. Table 1 contains the Lyapunov spectra for each of the experimental and surrogate time series. For the RT experimental data, the maximum Lyapunov exponent is positive, and the sum of the Lyapunov exponents is negative, suggesting that chaos might be present. Although the sum was negative for the surrogate data, the maximum Lyapunov exponent was also negative, suggesting different dynamics for the experimental and the surrogate series.

For the handwriting velocity time series, the results are a little more ambivalent, with the maximum Lyapu-

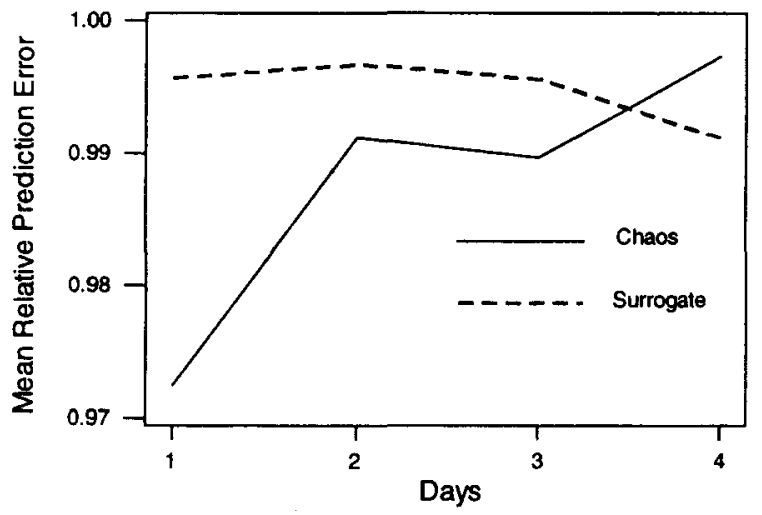

Figure 13. Mean relative prediction error for the chaotic (solid line) and phase-randomized surrogate (dotted line) conditions in a time series prediction experiment. 
Table 1

Lyapunov Exponents ( $\lambda 1$ to $\lambda 4$ ) for the Response Time (RT) and Handwriting (HW) Velocity Data for Both the Noise-Reduced Experimental (Exp) and the Surrogate (Surr) Time Series

\begin{tabular}{ccrcccc}
\hline Task & Series & \multicolumn{1}{c}{$\lambda 1$} & \multicolumn{1}{c}{$\lambda 2$} & $\lambda 3$ & $\lambda 4$ & Sum \\
\hline RT & Exp & 0.16 & -0.03 & -0.44 & -0.91 & -1.22 \\
RT & Surr & -0.35 & -0.71 & -1.23 & -1.45 & -3.74 \\
HW & Exp & 0.06 & -0.07 & -0.32 & -1.65 & -1.98 \\
HW & Surr & 0.01 & -0.04 & -0.32 & -2.23 & -2.58 \\
\hline
\end{tabular}

Note-A feedforward neural network with four input units, six hidden units, and one output unit was used to estimate the exponents.

nov exponents being both small and scarcely positive. In each case, the sum of the Lyapunov exponents was negative. In view of the small difference between the prediction functions for the noise-reduced and the surrogate handwriting velocity data, it is unlikely that this handwriting velocity time series is chaotic, although clear evidence for chaos in other examples of such series was obtained by Longstaff and Heath (1999).

\section{Conclusions}

Several techniques for analyzing the linear and nonlinear dynamics in experimental time series have been presented. These techniques include the linear techniques of autocorrelation and power spectral analyses and several nonparametric procedures for detecting nonlinearity, such as the time asymmetry and prediction tests, together with parametric procedures, such as recurrence plots, with their associated indices, and finally Lyapunov spectra.

Following analysis of three empirical data sets involving typing interkeypress times, sequential RTs, and horizontal handwriting velocity, it was shown that the typing data were unlikely to be nonlinear, since the time reversal statistic was not significant. On the other hand, the RT and handwriting velocity data exhibited some nonlinear determinism. In particular, the noise-reduced RT series produced more determinism in recurrence plots, lower prediction errors, and a positive largest Lyapunov exponent, when compared with the same quantities estimated from a surrogate series. These observations suggested that the RT series was probably chaotic. By contrast, the handwriting series demonstrated very little difference between the experimental and the surrogate sequences in both the prediction test and the Lyapunov spectra, suggesting that the dynamics may exhibit limit cycle behavior, rather than chaos, for this particular sample.

Once a quantification of the dynamics of a nonlinear system has been achieved, the next logical step is to determine what the underlying mathematical equations might be. This is a difficult task because, as we have seen, experimental data are always noisy and it is not easy to remove such contamination completely. Nevertheless, it is sometimes possible to estimate functions that relate the inputs (stimuli) and outputs (responses) of nonlinear systems, using neural networks and nonlinear system identification. Heath (2000) uses the latter technique to predict human performance in a variety of cognitive tasks, using nonlinear equations estimated from the relationships between stimuli and responses. Such technologies have wide potential application in many areas of psychology.

The use of surrogate data provides a convenient statistical hypothesis tool for detecting nonlinear determinism. The technology was used to design appropriate experiments for detecting human sensitivity to chaos in time series. It is inevitable that psychological data will be analyzed more frequently with nonlinear dynamical methods, once psychologists become more familiar with the technology. The Appendix contains World-Wide Web resources for analyzing nonlinear data sets.

\section{REFERENCES}

Abarbanel, H. D. I. (1996). Analysis of observed chaotic data. New York: Springer-Verlag.

GenÇAY, R., \& DEChERT, W. D. (1992). An algorithm for the $n$ Lyapunov exponents of an $n$-dimensional unknown dynamical system. Physica D, 59, 142-157.

GotTMan, J. M. (1981). Time-series analysis: A comprehensive introduction for social scientists. Cambridge: Cambridge University Press.

Grassberger, P., Hegger, R., Kantz, H., Schaffrath, C., \& SCHREIBER, T. (1993). On noise reduction methods for chaotic data. Chaos, 3, 127-141.

Heath, R. A. (1998). From simple processes does complex behavior emerge: A methodology for cognitive research based on nonlinear systems theory. In J. Wiles \& T. Dartnall (Eds.), Perspectives on cognitive science: $I I$ (pp. 15-45). Stamford, CT: Ablex.

Heath, R. A. (2000). Nonlinear dynamics: Techniques and applications in psychology. Mahwah, NJ: Erlbaum.

Heath, R. A., \& Willcox, C. H. (1990). A stochastic model for interkeypress times in a typing task. Acta Psychologica, 75, 13-39.

HegGer, R., Kantz, H., \& Schreiber, T. (1999). Practical implementation of nonlinear time series methods: The TISEAN package. Chaos, 9, 413-435.

HoWELL, D. C. (1997). Statistical methods for psychology. Belmont, CA: Duxbury.

Kantz, H., \& SChreiber, T. (1997). Nonlinear time series analysis. Cambridge: Cambridge University Press.

KAPLAN, D., \& Glass, L. (1995). Understanding nonlinear dynamics. New York: Springer-Verlag.

KAUFFMAN, S. A. (1993). The origins of order: Self-organisation and selection in evolution. New York: Oxford University Press.

Kelly, A., Heathcote, A., Heath, R., \& Longstaff, M. (in press). Evidence for linear and nonlinear dynamics in human reaction time sequences. Journal of Experimental Psychology.

LongstafF, M. G., \& Heath, R. A. (1999). A nonlinear analysis of the temporal characteristics of handwriting. Human Movement Science, $18,485-524$.

MetzGer, M. A. (1994). Have subjects been shown to generate chaotic numbers? Commentary on Neuringer and Voss. Psychological Science, 5, 111-114.

Metzger, M. A., \& Theisz, M. F. (1994). Forecast: Program to obtain forecasts from subjects for successive values of chaotic time series. $B e$ havior Research Methods, Instruments, \& Computers, 26, 387-394.

Neuringer, A., \& Voss, C. (1993). Approximating chaotic behavior. Psychological Science, 4, 113-119.

SCHREIBER, T. (1999). Interdisciplinary application of nonlinear time series methods. Physics Reports, 308, 1-64.

SMITHSON, M. (1997). Judgment under chaos. Organizational Behavior \& Human Decision Processes, 69, 59-66.

Webber, C. L., JR., \& Zbilut, J. P. (1996). Assessing deterministic structures in physiological systems using recurrence plot strategies. In M. C. K. Khoo (Ed.), Bioengineering approaches to pulmonary physiology and medicine (pp. 137-148). New York: Plenum. 


\section{APPENDIX \\ List of Some World-Wide Web Sites Offering Nonlinear Data Analysis Software}

Because of space limitations, readers are advised to check the accompanying World-Wide Web sites for detailed information on the availability of nonlinear techniques, together with the latest pricing and ordering information.

\section{TISEAN 2.0}

This free package is perhaps the most authoritatively documented of all the presently available noncommercial ones. It contains a comprehensive range of procedures for analyzing all aspects of linear and nonlinear data sets. The only disadvantage is its DOS/UNIX interface and the lack of a user friendly Windows environment. http://www.mpipks-dresden.mpg.de/ tisean/ TISEAN_2.0/index.html

\section{Santis/Dataplore}

Although previously freeware, this Windows-based, reasonably comprehensive, and usable package is now available only commercially. It contains a large array of linear and nonlinear data analysis techniques. It costs $\$ 1,099$ for the full system, available for Windows NT and UNIX. http://www.datan.de/dataplore/

\section{Chaos Data Analyzer}

Perhaps the classic nonlinear analysis package containing a variety of nonlinear data analysis procedures, all of which are accompanied by readily interpreted graphics visualization. The package suffers from inflexibility and some documentation problems, especially for the computational algorithms used. Nevertheless, it is very useful for classroom demonstration purposes and data analyses. The Professional Version (recommended) costs $\$ 300$ for a single license. http://sprott.physics.wisc.edu/ cda.htm

\section{Visual Recurrence Analysis}

This nicely designed free package uses a friendly Windows interface to construct recurrence plots and compute various nonlinear indices. http://pweb.netcom.com/ eugenek/download.html

\section{NETLE Software Package}

This free program uses a feed-forward neural network to estimate a nonlinear function predicting the next time series value from a small set of preceding values. The derivatives of this function are used to estimate the Lyapunov spectrum. http://www.bsu. edu/econ/tliu/download/index.html

\section{Tools For Dynamics}

A professional nonlinear dynamics analysis package for UNIX and Windows environments, with a comprehensive array of data analysis and visualization features. The UNIX version costs $\$ 1,495$, a Windows version sells for $\$ 845$, and a version for Macintosh can be obtained for $\$ 495$. http://www.zweb.com/apnonlin/

(Manuscript received November 1, 1999; revision accepted for publication March 9, 2000.) 